\title{
Review
}

Dongdong Huang, Ke Wu, Ying Zhang, Zhihui Ni, Xiaohong Zhu, Can Zhu, Jianjing Yang*, Qichuan ZhuGe*, and Jiangnan $\mathrm{Hu}^{*}$

\section{Recent Advances in Tissue plasminogen activator-based nanothrombolysis for ischemic stroke}

https://doi.org/10.1515/rams-2019-0024

Received Mar 01, 2019; accepted Jul 21, 2019

\begin{abstract}
Stroke is an acute cerebrovascular disorder caused by sudden decrease or interruption of blood flow in brain arteries. Deficiency of timely and effective reperfusion of ischemic brain tissue can lead to irreversible brain injury and neurological dysfunction. Currently, recombinant tissue plasminogen activator (rt-PA) is the only appropriate thrombolytic agent which is approved by FDA for patients with acute ischemic stoke. However, due to the limitation of very narrow therapeutic time window and severe intracranial hemorrhagic complication, the outcome of stroke treatment mediated by rt-PA still remains unsatisfactory. Therefore, it is urgent to find new alternative drugs or develop novel drug delivery system to achieve better outcomes. In recent years, with the rapid development of nanotechonology, nanomaterials as a drug delivery system can provide new strategies and methods to carry tPA specifically to the occlusion site and provide advanced treatment for stroke. In this review, we briefly introduced the physiopathologic mechanisms of thrombolysis and focused on the comparison of the t-PA mediated thrombolysis and t-PA conjugated nanomaterial mediated thrombolysis.
\end{abstract}

Keywords: Tissue plasminogen activator, Drug delivery, Nanomaterial, Stroke, Thrombolysis

\footnotetext{
*Corresponding Author: Jianjing Yang: Department of Neurosurgery, The First Affiliated Hospital of Wenzhou Medical University, Wenzhou 325000, China; Zhejiang Provincial Key Laboratory of Aging and Neurological Disorder Research, The First Affiliated Hospital of Wenzhou Medical University, Wenzhou 325000, China; Email: yangjianjing2@163.com

*Corresponding Author: Qichuan ZhuGe: Department of Neurosurgery, The First Affiliated Hospital of Wenzhou Medical University, Wenzhou 325000, China; Zhejiang Provincial Key Laboratory of Aging and Neurological Disorder Research, The First Affiliated Hospital of Wenzhou Medical University, Wenzhou 325000, China, Email: zhugeqichuan@vip.163.com
}

\section{Introduction}

Stroke has become the primary fatal and first disabling disease after malignant tumor in the worldwide [1, 2]. It is an acute cerebrovascular disease characterized by cerebral blood circulation disorders which is caused by sudden intracerebral artery stenosis, occlusion or rupture due to various factors. Insufficient blood flow and oxygen in the blood vessels lead to irreversible brain injury, severe cognitive, motor impairments and even death [3, 4]. About $75 \%$ of the stroke survivors have suffered from different degrees of neurologic deficits, leading to permanent disability, and greatly impacting patients' quality of life [5]. According to the Global Burden of Disease study statistics in 2017, the incidence of disability-adjusted life-years caused by stroke accounts for more than 5\%, ranking second only next to ischemic heart disease and neonatal diseases [6].

There are two types of stroke based on the differences of physiopathologic mechanism, ischemic stroke and hemorrhagic stroke. Ischemic stroke accounts for about $70 \%$, remains to be the common type which occurs as a result of an obstruction by a clot or a mass within an brain artery [7]. Ischemic stroke is classified into two major subtypes, thrombotic and embolic types, which occur on the base of thrombus formed on the atherosclerotic plaque

*Corresponding Author: Jiangnan Hu: Department of Neurosurgery, The First Affiliated Hospital of Wenzhou Medical University, Wenzhou 325000, China; Zhejiang Provincial Key Laboratory of Aging and Neurological Disorder Research, The First Affiliated Hospital of Wenzhou Medical University, Wenzhou 325000, China; Department of Pharmacology and Neuroscience, University of North Texas Health Science Center, Fort Worth, Texas 76107, United States of America; Email: hu.jiangnan@hotmail.com

Dongdong Huang, Ke Wu, Ying Zhang, Zhihui Ni, Xiaohong Zhu, Can Zhu: Department of Neurosurgery, The First Affiliated Hospital of Wenzhou Medical University, Wenzhou 325000, China; Zhejiang Provincial Key Laboratory of Aging and Neurological Disorder Research, The First Affiliated Hospital of Wenzhou Medical University, Wenzhou 325000, China 
and thrombus originated outside the brain, respectively [4, $8,9]$.

Previous study has shown that an average of 1.9 million brain cells die per second when the blood flow supplying to brain tissue is completely disrupted [10]. However, as cerebral vessels are rarely completely blocked in acute ischemic stroke, residual perfusion still exists in the ischemic region due to the effect of local perfusion pressure and collateral circulation [11, 12]. This particular region was first defined by Astrup et al. [11] in 1981 and is known as the ischemic penumbra, a circular region between the dense center of ischemic necrosis area and the normal brain tissue. They demonstrated that hypoperfused brain tissue has the capacity to recover if perfusion is improved. The most critical factors to achieve better final outcome of stroke are to maintain or improve the residual perfusion of acute focal ischemia and restore perfusion of penumbra as early as possible $[11,13]$. During the therapeutic window of saving penumbra, every 1 min reduction of disease progress can achieve a better neuroprotection and improvement of clinical prognosis of penumbra by increasing of 1.8 days in the lifetime without disability [14]. Current management of stroke starts with rapid and accurate assessments, comprehensive admission protocols, early specialist management in a stroke unit, and thrombolysis strategies for acute ischemic stroke, along with early use of antiplatelet drugs and appropriate physiological monitoring [15]. Timely and effective thrombolysis therapy becomes the most important intervention that can potentially reduce disability and effectively improve the outcomes of acute ischemic stroke. Currently, the clinical strategies for achieving recanalization of occluded vessels mainly include intravenous thrombolysis, intra-arterial thrombolysis, mechanical thrombectomy and stent angioplasty [16-19]. To date, the intravenous thrombolytic therapy for acute ischemic stroke has been mainly selected in consideration of the thrombolytic time window. Intravenous injection of tissue plasminogen activator ( $\mathrm{t}-\mathrm{PA}$ ) has been proven to be valid pharmacological treatment for regain of blood flow and it is the only thrombolytic drug approved by the food and drug administration (FDA) for the treatment of acute ischemic stroke [20]. Unfortunately, treatment of t-PA may lead to a severe and deadly complication-symptomatic intracerebral hemorrhage, caused by a variety of mechanisms. Either combined IPA, degraded LRP1 or active plateletderived growth factor-CC (PDGF-CC) would markedly increase the permeability of $\mathrm{BBB}$, which was considered to be one of the most important mechanisms that causes intracerebral hemorrhage [21]. Its narrow therapeutic time window and severe complications limit its wider applica- bility immensely [20]. Based on the result of seven different doses used in the National Institute of Neurological Disorders and Stroke (NINDS) trial in 1995, t-PA is recommended to use within $3 \mathrm{~h}$ after the onset of stroke symptoms. This time window was not altered until 2008, a randomized controlled trial named The Third European Cooperative Acute Stroke Study administered alteplase 3-4.5 $h$ since the onset of stroke found that compared to the control group patients were significantly to have a favorable outcome in the group treated with alteplase [22]. The risk of symptomatic intracerebral haemorrhage (sICH) in patients treated with alteplase, though, was significantly greater than those treated with placebo, but the rate was obviously less than the previous trials those applied alteplase within $3 \mathrm{~h}$ once symptoms onset. Meanwhile, a prospective observational study called Safe Implementation of Treatments in Stroke-International Stroke Thrombolysis Registry (SITS-ISTR) indicated no significant difference in excellent recovery, functional independence, sICH and mortality between the patients treated with alteplase 3-4.5 $\mathrm{h}$ and $3 \mathrm{~h}$ of symptoms onset [23]. Ultimately, these two innovative studies provided vital evidence to support expanding the limited therapeutic time window. European Stroke Organization (ESO), National Stroke Foundation of Australia and American Heart Association and American Stroke Association etc. issued updated guidance to adopted the extended time window for thrombolysis of 4.5 $\mathrm{h}$ subsequently $[16,24,25]$. In addition to some patients, prolonged t-PA treatment window may increase the risk of cerebral hemorrhage [26]. One clinical study based on 3,035 peoples (IST-3) showed that intravenous injection of t-PA within 6 hours of stroke can significantly increase the incidence of intracranial hemorrhage within 7 days [27]. Despite the specific efficacy and safety of rt-PA in intravenous thrombolysis within therapeutic time window, only $2.4 \% \sim 5.2 \%$ of acute ischemic stroke patients are able to get access to intravenous thrombolysis due to a delayed treatment time or other various reasons [28]. In recent years, endovascular therapy as an alternative treatment has been developed greatly and increased the rate of occlusive vascular recanalization significantly $[17,18]$. However, some studies have pointed out that endovascular therapy puts forward higher requirements for the construction of highly specialized centers and the professional skills of relevant medical personnel. The high professional requirements for hospital and doctor restrict the extensive application of this procedure, especially in rural and remote areas $[29,30]$. Thus, it is urgent to find more safe and effective thrombolytic techniques and methods to solve the problems of vascular recanalization and blood flow reconstruction in ischemic stroke. 


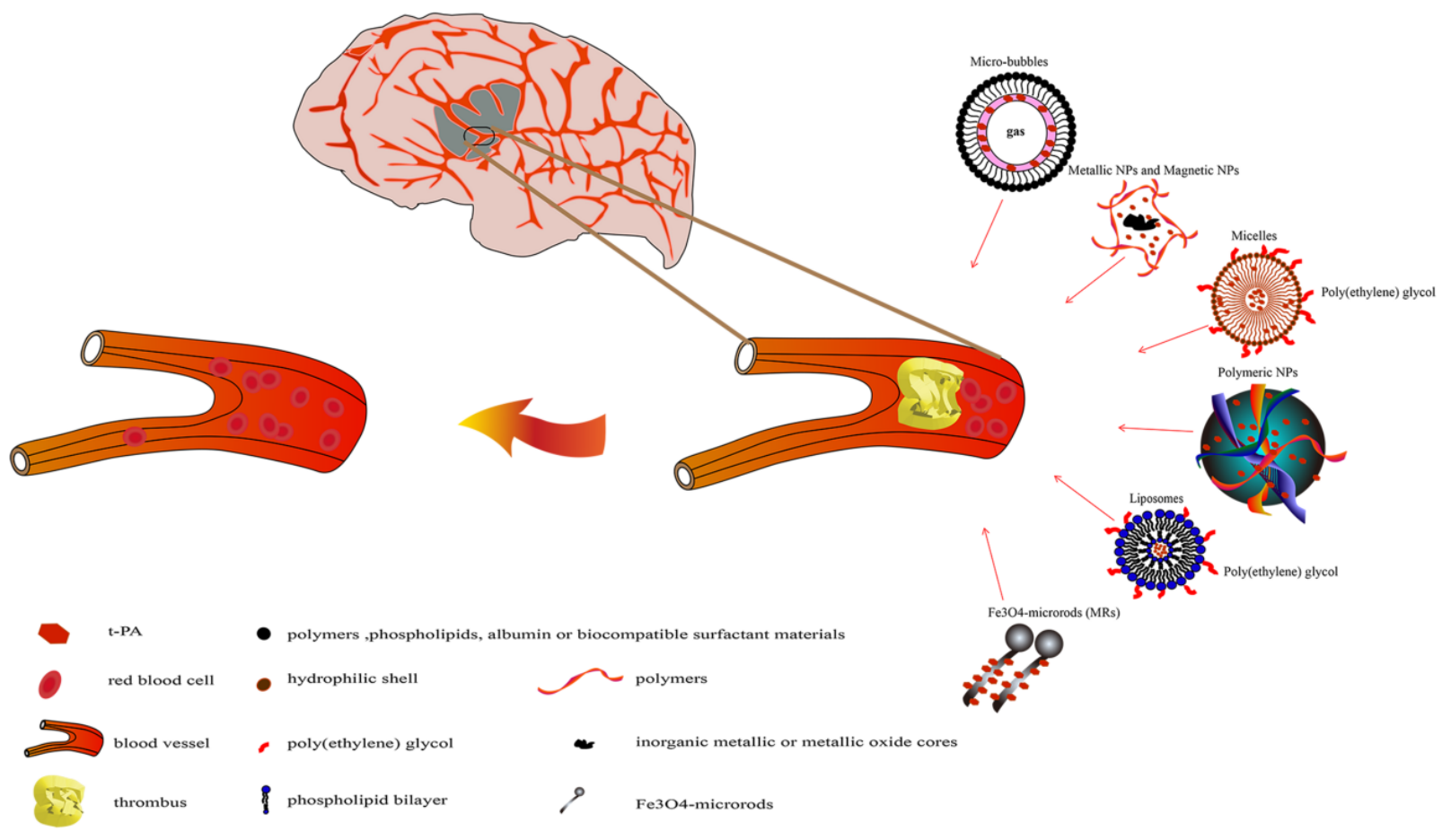

The sketch of various t-PA conjugated nanomaterials for improved thrombolysis

Figure 1: Ischemic stroke presents ischemia and hypoxia of local tissue due to the lose of blood flow to the distal vessels by clot. The application of various t-PA conjugated nanomaterials can achieve more effective thrombolysis effects and faster vascular recanalization compared to t-PA only.

Put together, with the development of nanomedicine technology, the application of nanoparticles as carriers to deliver t-PA to the thrombus sites for efficient thrombolysis has brought new hope for the treatment of stroke [31]. The major purpose of this article is to review the current nanomaterial applications for acute ischemic stroke, mainly focused on the comparison of t-PA-mediated therapeutic effects and the one facilitated by various novel nanomaterials (Figure 1). Moreover, we further discussed the bottlenecks and potential future directions of nanothrombolysis in stroke treatment.

\section{2 t-PA-mediated thrombolysis for ischemic stroke}

In 1902, t-PA was identified and named fibrikinase by Conradi on the first time. Collen et al. [32] purified the t-PA in larger amounts from Bowes melanoma cell line and 10 the primary structure of t-PA was provided when Pennica et al. [33] succeeded in cloning and expressing recombinant t-PA. The targeted fibrinolytic function of t-PA for myocardial infarction was successfully exploited in the 1980s and investigators applied t-PA to ischemic stroke research subsequently [34]. t-PA is a serine protease of 527 or 530 aminoacids including three glycosylation sites and 17 disulfide bridges [35]. A mosaic protein that consists of five distinct modules: a finger domain (F), an epidermal growth factorlike domain(EGF), two kringle domains (K1 and K2), and a serine protease proteolytic domain (SP) constitutes the mature form of t-PA. Different domains bind different proteins, substrates and receptors to mediate different functional activities of t-PA. For example, the finger domain is involved in its ability to cross the blood-brain barrier (BBB) [36] and the lysine binding site (LBS) in K2 domain is contributed to the capacity of t-PA to bind and activate plasminogen and NMDAR(N-methyl-D-aspartate receptor) [37]. Under the effect of kallikrein or plasmin, the secreted single-chain tPA (sc-tPA) can be processed into a two-chain form tPA (tctPA). However, since the activation of sc-tPA depends on the allosteric regulator, such as fibrin. sc-tPA could show the same catalytic activity with tc-tPA under fibrin condition, otherwise the efficiency of former t-PA will be dramatically reduced [38].

According to different N-glycosylation sites, plasminogen activators were divided into two categories: type I t-PA's glycosylation sites locate at Asn117, Asn184 and 
Asn448, and type II t-PA is only at Asn117 and Asn448 [35]. Depending on different sources, t-PA can also be classified into two types: endogenous t-PA and exogenous t-PA. Endogenous t-PA is confirmed to be mainly synthesized and released by vascular endothelial cells, and also has a degree of expression in the brain which contains hippocampus, amygdala, cerebellum, and hypothalamus [39]. All the thrombi are consisted of aggregated platelets and fibrin monomers. The thrombi origin from patients with cardioembolic stroke are fibrin-rich [40], while thrombi from patients with non-cardioembolic strokes mainly composed of red blood cells rather than platelets and fibrin [41]. Once the thrombi form, t-PA will bind to lysine binding site of plasminogen in the bloodstream and fibrin exposed on the surface of clot due to the high binding specificity mentioned above. Thereupon the ternary complex contains plasminogen, t-PA and fibrin is constructed successfully, which can catalyze the conversion of inactive plasminogen to active serine plasminase, thus initiate the fibrinolysis system [42]. Subsequently, occluded blood vessel realize recanalization with the action of plasmin crosslinked fibrin be massively lysised into fibrin degradation products [42]. PAI-1 is demonstrated to covalently bind to the C-terminal catalytic domain of t-PA, forming a PAI-1/ t-PA complex which can be degraded in liver through lowdensity lipoprotein receptor-related protein-1 (LRP-1) mediated pathway [43], and ensure the coagulation and fibrinolysis system in a dynamic balance. Despite of t-PA's primary function in thrombolysis, t-PA has also been reported as deleterious or beneficial for neuronal function in patients with acute ischemic stroke. These nonfibrinolytic functions are difficult to be reconciled, while some researchers proposed it to be neuroprotective, and others considered it to be neurotoxic [35]. Many studies indicated that receptors of neurons such as NMDAR, LRP-mediated PSD95 activation, and EGF receptor displayed a key role in the prosurvival and pro-neurotoxic effects of t-PA [44-46]. For instance, Montagne et al. [47] proposed that exogenous t-PA could promote neurotoxicity on cortical neurons via activating extrasynaptic GluN2D-containing NMDARs. On the contrary, Wu et al. [48] pointed out that exogenous t-PA could produce a neuroprotective effect through synaptic GluN2A-containing NMDARs. t-PA generated in the brain activates matrix metalloproteases (MMPs) in the brain especially MMP-9 to degrades extracellular matrix components [49]. Therefore, BBB in which the normal function of cell to cell interactions are maintained by collagen and fibronectin will be damaged. Accordingly, increased permeability of BBB will cause a series of complications, including cerebral edema, intracerebral hemorrhage and infarct extension $[50,51]$.
By contrast, Haile et al. [49] suggested that t-PA's neuroprotective effect on neurons which may mainly relies on antiapoptotic effects by using transgenic mice over-expressing t-PA in neurons. The advantages and disadvantages of t-PA for neurons are time-dependent, regional related and involve variety of complex mechanisms. Roughly, the pro-survival effects of t-PA are mediated by EGF receptors, annexin II, PI-3 kinase-, AMPK-, mTor-HIF1alpha-dependent signaling pathways, and the neurotoxic effects of t-PA are considered to be related to its proteolytic activity, targeting plasminogen, NMDARs, components of the extracellular matrix and inflammatory mediators $[35,52]$. According to the criteria used in the landmark NINDS trial in which patients treated with t-PA within three-hours of the onset of symptoms achieved an absolute improvement of $15 \%$ in excellent outcomes and no significant increase in mortality, $3 \mathrm{~h}$ was regarded as therapeutic time window for t-PA in patients with stroke [20]. The remarkable clinical benefit of t-PA is inspiring, however, concomitant a threefold increase in the risk of sICH is frustrating. Since the NINDS, a number of further randomized controlled trials aiming to extend the time window for t-PA beyond three-hours from symptoms onset, but the majority of them end in failure [53-55]. However, a pooled analysis [56] included several trials such as NINDS, European Cooperative Stroke Study (ECASS) I, ECASS II trials and ATLANTIS, showed a potential benefit up to $4.5 \mathrm{~h}$ after the onset of symptoms. According to positive results of this meta-analysis, the third ECASS III and SITS-ISTR were promoted and the extended time window was once again verified [22, 23]. In several superiority trials, IV tenecteplase within $3 \mathrm{~h}$ has been compared to IV alteplase up to 6 hours after stroke onset; safety of tenecteplase shows to be similar to alteplase, whiles the effective comparison remains unclear $[57,58]$. As benefit from therapy is considered to be time dependent, IV alteplase should be initiated as quickly as possible in eligible patients. sICH is the major adverse effect of thrombolysis, with an incidence of $6-7 \%$. In a Cochrane Review of clinical trials, sICH occurred in 60 out of every 1,000 patients treated [odds ratio (OR) 3.28, $95 \%$ CI 2.484.33, $\mathrm{p}<0.00001$ ] [59]. The occurrence of sICH is associate with age, high blood pressure, severe neurological deficits and hyperglycaemia [60]. Because of the limited therapeutic window, deficient awareness of early symptom and excessive concern about ICH, only less than $5 \%$ of stroke patients have access to rt-PA treatment $[55,61]$. rt-PA is a mutant formed by the deletion of structural domains, glycosylation modification or amino acid substitution of natural t-PA as a template through genetic engineering, which can get a long plasma half-life, resist natural inhibitors, and enhance the binding force and specificity of 
fibrin [62]. Currently, alteplase for injection is the only second generation of rt-PA that approved by FDA for thrombolysis in acute ischemic stroke. The plasma half-life of alteplase is very short, only 4-6 mins, and continuous intravenous infusion is required after the first intravenous injection. NINDS [20] results suggested that the dose of alteplase was $0.9 \mathrm{mg} / \mathrm{kg}$ bodyweight, with a single maximum dose of $90 \mathrm{mg}, 10 \%$ of the total dose was first injected intravenously, and the rest was intravenous drip within 60 mins. A review study found there are significant improvements in neurological function in stroke patients treated with alteplase compared with those treated with placebo (mRS $0 \sim 152.4 \%$ vs 45.2\%, OR=1.34, 95\%CI: $1.02 \sim 1.76, \mathrm{P}<$ 0.05) [63]. The incidence of symptomatic intracranial hemorrhage was significantly higher in the treatment group $(2.4 \%)$ than in the placebo group (0.2\%).

Reteplase is the third generation of recombinant mutant with finger domain (F), epidermal growth factor-like domain (EGF) and kringle domain deleted. Although its affinity with fibrinin was four times lower than that of alteplase, its half-life was extended from 4 mins to 15 mins. The thrombolytic effect of reteplase was significant, however, it also leads to the bleeding adverse reaction. To date, there is no large randomized, double-blind controlled study of reteplase and it has not been FDA approved for use in acute ischemic stroke [64].

Tenecteplase is another kind of variant with modification at three site T103N, N117Q and KHRRR(296-299)AAAA [64]. The fibrous specificity of teneptidase is higher than alteplase and reteplase, and it has a stronger affinity for blood clots rich in fibrinogen, but its catalytic effect on plasminogen activation is weaker than alteplase. FDA approval of tenecteplase for thrombolytic therapy of acute myocardial infarction was $0.5 \mathrm{mg} / \mathrm{kg}$ intravenous infusion. In a study including 88 patients for the application of tenecteplase, the main improvement in neurological function evaluated at $24 \mathrm{~h}$ and $90 \mathrm{~d}$ was significantly better than the placebo group in NINDS [65]. Unfortunately, the trial was terminated due to sICH in 2 of the 13 patients in the $0.5 \mathrm{mg} / \mathrm{kg}$ group. Moreover, Parsons et al. [58] conducted a randomized controlled clinical trial among the standard dose of alteplase, tenecteplase $0.1 \mathrm{mg} / \mathrm{kg}$ and tenecteplase $0.25 \mathrm{mg} / \mathrm{kg}$. After the combination of two doses of tenecteplase, the main endpoint events, such as clinical improvement and CT reperfusion at $24 \mathrm{~h}$ were all significantly better than that of alteplase group. The proportion of patients with good recovery (mRS score $0 \sim 2$ ) at $90 \mathrm{~d}$ was also significantly higher than that in the alteplase group.

Desmoteplase, derived from vampire bat saliva, with only a kringle domains and no lysine binding site, showed safety and efficacy in pilot studies [66, 67]. Subsequent Phase III DIAS-2 study found no benefits within 90-day clinical outcomes in both dose group, consistent incidence of ICH and increased mortality in the high-dose group (21\% versus $5 \%$ low dose; $6 \%$ placebo [68]. This result may be related to the relatively mild disease of the selected patients, resulting in the high clinical improvement rate in the placebo group at 90 days of follow-up. Whether desmoteplase could become a promising agent for acute ischemic stroke thrombolysis treatment relies on phase III clinical study.

\section{Novel nanomaterials and t-PA conjugated nanomaterials for thrombolysis}

Furthermore, several previous studies have shown that exogenous t-PA can increase the permeability of neurovascular unit in human stroke patients $[69,70]$, as well as the permeability of the BBB [71]. Hence, the capacity of t-PA to modulate BBB opening was proposed to be relevant to the ischemic cerebral hemorrhage following its administration to patients with ischemic stroke [72]. Moreover, tPA can also exert neurotoxic effects on brain tissue in ischemic regions by penetrating damaged BBB. Considering the narrow therapeutic time window, intracranial hemorrhage and neurotoxic side effects, establishing new strategies to increase thrombolysis efficiency and decrease the dose of thrombolytic drugs are urgently needed. Early diagnosis and prevention of stroke, as well as drug/RNA delivery by using various types and sizes of nanomaterials to acquire improved treatment and neuroprotection have been wildly reported in this field [73]. Nanomaterial, a novel material possessing unique physiochemical properties [74] and excellent cytocompatibility properties has provided new promising solutions for stroke. In addition, the stability of nanomaterials allows them to bind to specific ligands, thereby enhancing targeted therapeutic effects [73]. With the advantages of accurate dose quantify by nanosynthesis and exact targeting by dynamic delivery system, nanocarrier drug delivery has become the uppermost form of application in stroke. $t$-PA-conjugated nanoparticles are considered to decrease the risk of intracerebral hemorrhage (ICH) by restraining t-PA activity in the circulation provisionally. Due to the very short half-life of t$\mathrm{PA}$, more than $50 \%$ of t-PA is cleared within five-minutes of delivery, the encapsulation of t-PA by nanoliposomes can help to prolong its half-life in the peripheral circulation 


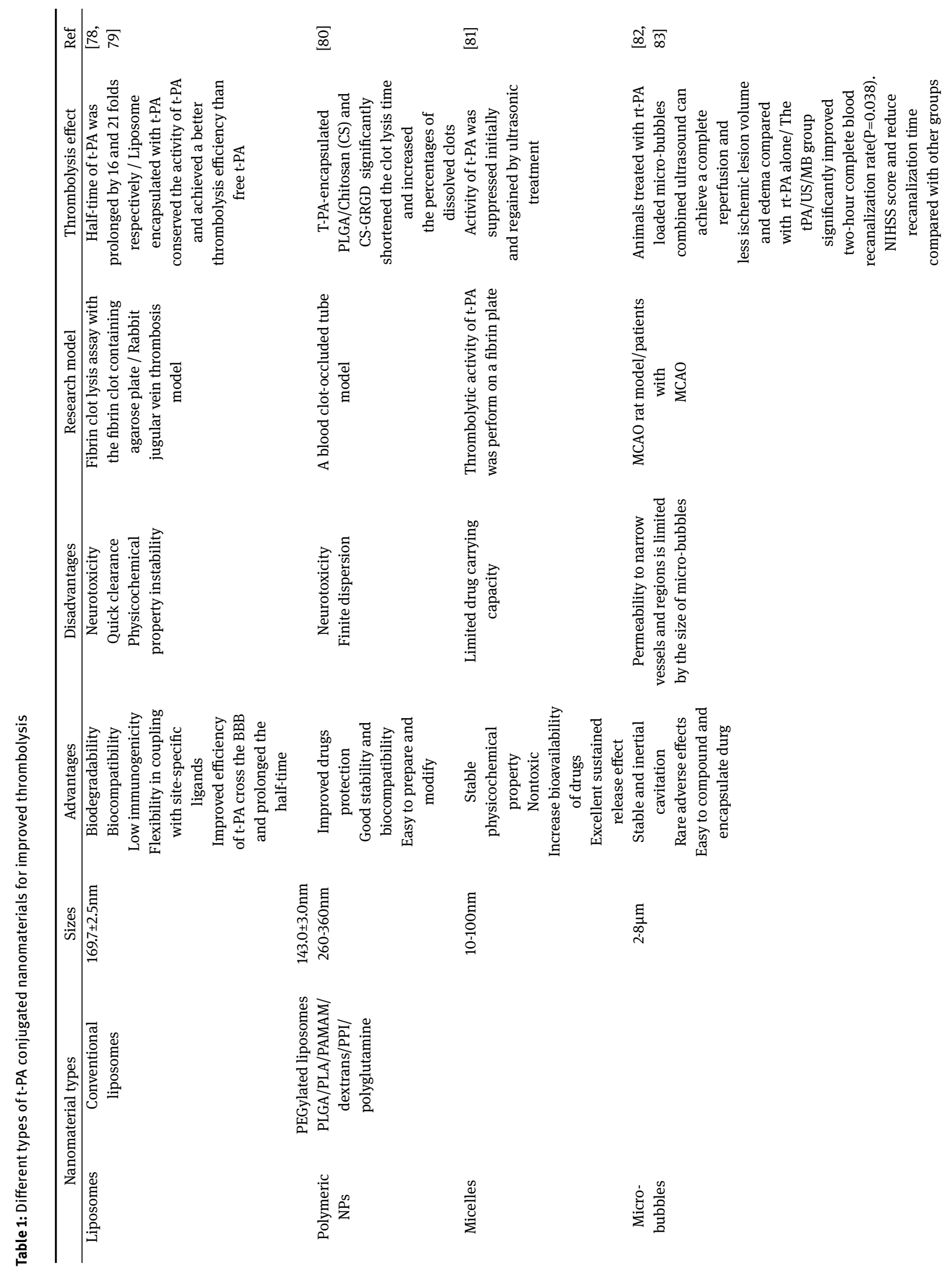




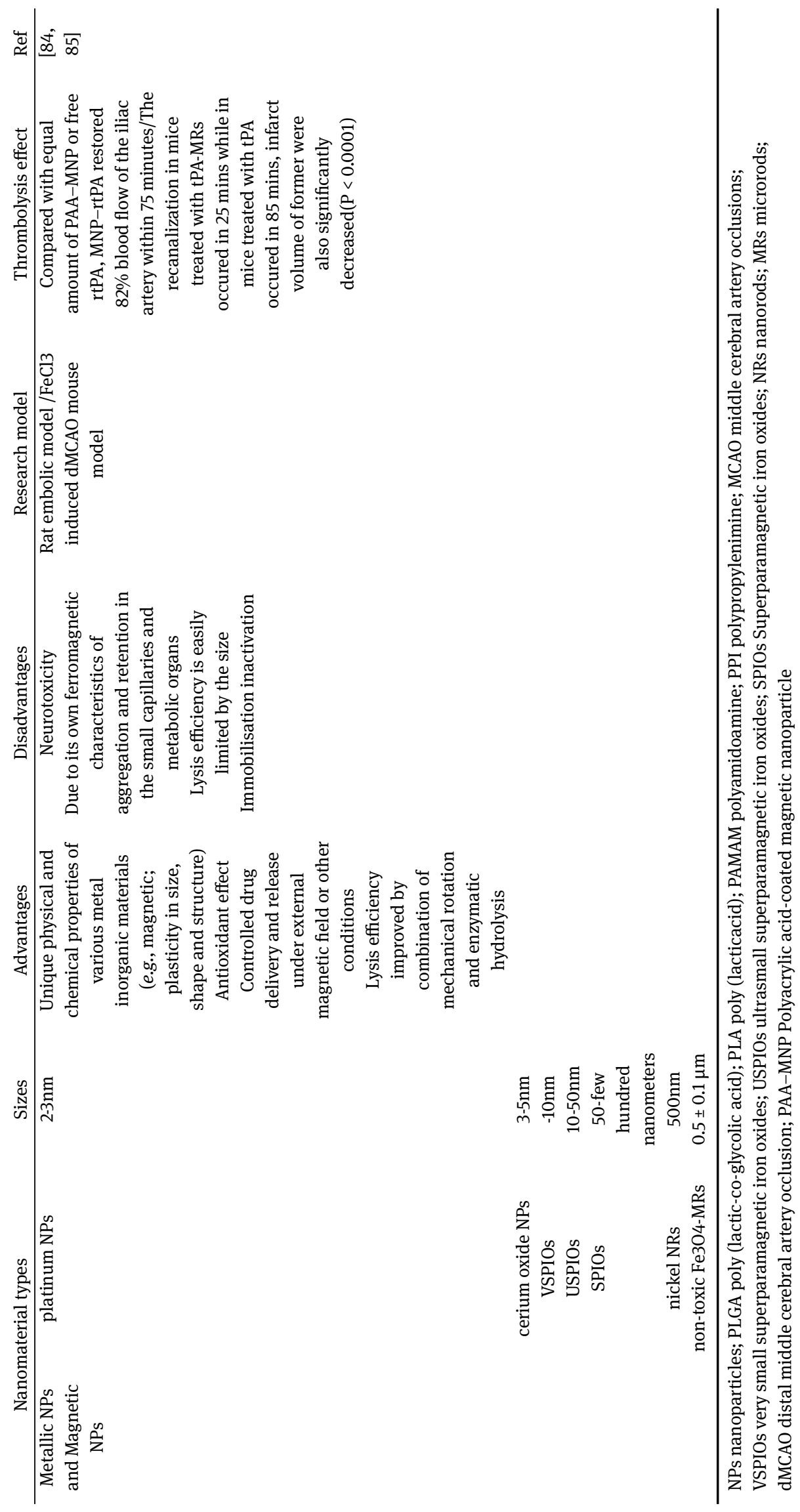


[75] and reduce the potential risk of t-PA-mediated hemorrhage at non-target regions. With the combination of mechanical and chemical properties, tPA-conjugated nanoparticles can also promote the infiltration of t-PA into deep thrombus to maximize the thrombolysis efficiency and achieve better reperfusion of occluded vessels within the shortest time. Examples of applied nanoparticles include liposomes, micelles, biodegradable polymeric nanoparticles (NPs), micro-bubbles, metallic NPs and magnetic NPs (Table 1). These novel materials have exhibited abundant benefits in reducing drug inactivation, improving pharmacokinetic profiles and enhancing targeted transportation of drugs [76, 77].

\subsection{Liposomes}

Liposomes are lipid bilayer arrays that consist of hydrophobic phospholipid bilayer and a hydrophilic aqueous core $[86,87]$. This structure allows liposomes to encapsulate both water-soluble and water-insoluble compounds [88]. With the advantages of biodegradability, biocompatibility, low immunogenicity, and flexibility in coupling with site-specific ligands, liposome was the earliest and widely used nanocarrier for drug delivery [89]. Heeremans and his colleagues have demonstrated that $\mathrm{t}$ PA-loaded liposomes can increase thrombolysis efficiency than free t-PA [78]. After chemically modified by polyethylene glycol (PEG) on the surface of liposome, its short halflife which is limited by phagocytosis of reticuloendothelial system could be significantly improved [90]. Ji-Young Kim et al. [91] found that half-life of t-PA encapsulated into conventional liposomes (EPCL) and PEGylated-liposomes (EPC-PEGL) could be enhanced from 5.87 minutes to 50.03 mins and 132.62 mins respectively.

\subsection{Micelles}

Micelles are polymers of amphiphilic molecules that have attracted attention as nanocarriers for drug delivery. The size of micelles ranges from 10 to $100 \mathrm{~nm}$ [92]. Hydrophilic shell exposed to the surrounding water and hydrophobic core can form micelles spontaneously in the aqueous solution. Hence, encapsulated drugs could be prevented from degradation, and their half-life in blood circulation time can also be increased [93]. In order to load multiple agents of different properties, the size, molecular composition and surface structure of micelles can be usually changed. For instance, PEG is commonly used to modify micelle, to get hydrophilic and capacity to escaping endocytosis from macrophages [77].

\subsection{Polymeric NPs}

Polymeric NPs are composed of several polymers such as poly (lactic-co-glycolic acid) (PLGA), poly (lacticacid) (PLA), polyamidoamine (PAMAM), dextrans, polypropylenimine (PPI) and polyglutamine. For example, biodegradable PLGA NPs are copolymer synthesized by lactic acid and opening cyclic dimers (1,4-dioxane-2,5diones) of glycolic acid rings with the catalytic action of tin (II) 2-ethylhexanoate, tin(II) alkoxides or aluminum isopropoxide [94]. Mdzinarishvili et al. [95] found that thyroid hormones (T3) combined with glutathione-coated PLGAbPEG NPs can significantly prevent brain from ischemic damage as compared with a solution of T3. Another study demonstrated that infarct volume and neurological deficit can be improved once transport tanshinone IIA into the brain by cationic bovine serum albumin conjugated PEGylated NPs efficiently [96]. In addition, release of drugs can be affected by the rates of polymer biodegradation and drug diffusion [97]. Furthermore, by endocytosis, this nanoparticle can also penetrate cell membranes and deliver therapeutic drugs into the cytoplasm.

\subsection{Micro-bubbles}

With a size between 2 to $8 \mu \mathrm{m}$, similar order of magnitude to red blood cell, microbubble is considered to be another promising kind of lipid based deliveries used in thrombolytic treatments [98]. Typical microbubbles consist of a shell compose of polymers, phospholipids, albumin or biocompatible surfactant materials, and a core filled with air or high molecular weight gas [98]. Coating microbubbles are known to be widely used as contrast agents in ultrasonic imaging in the clinic. Under the impact of acoustic field, gas-filled bubbles would occur volume oscillations which is called acoustic cavitation may paly an important role in the mechanisms of microbubble-mediated clot lysis. Multiple previous studies have showed that ultrasound combined with microbubble contains thrombolytic drugs or microbubble alone could improve the recanalization of obstructed vessels in animals [82,99]. In a rat model of autologous thrombus induced acute cerebral ischemia, Moumouh et al. [99] demonstrated that the restoration of occluded blood flow in rats treated with sulfur hexafluoride lipid based microbubbles associated with ultrasound was similar to those intravenous administration 

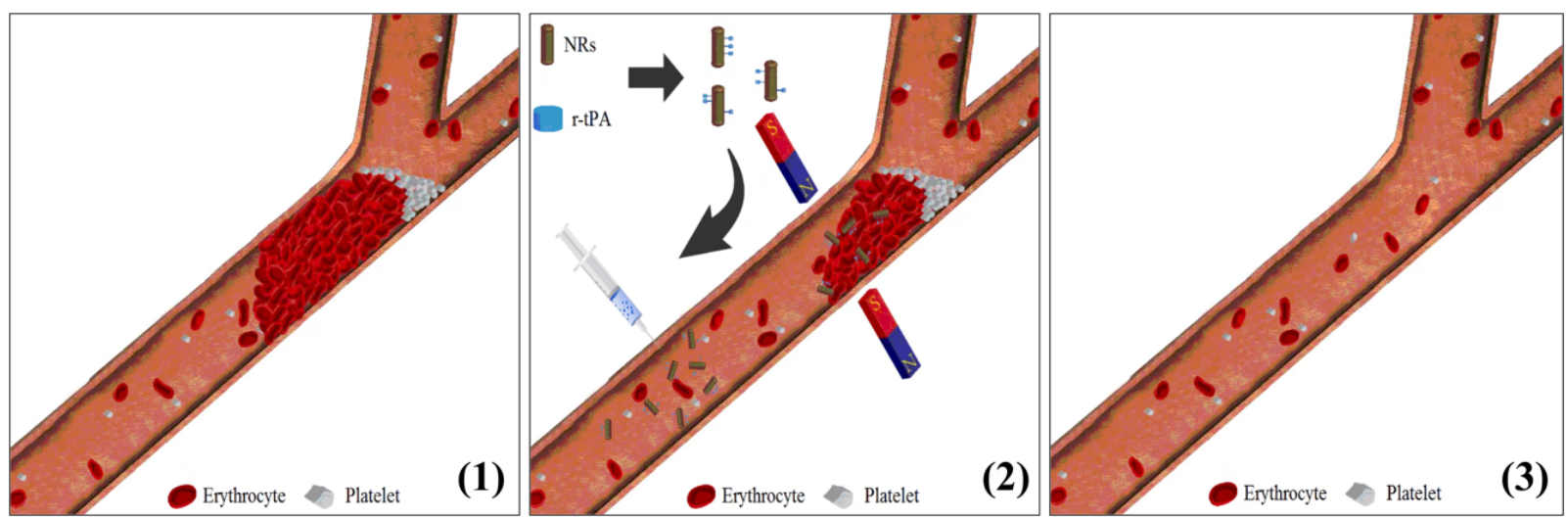

Figure 2: Schematic images of accelerated thrombolysis effects mediated by tPA-Fe $\mathbf{O}_{4}$-MRs. (1) Thrombus in the distal middle cerebral artery. (2) Synthesis of tPA-MRs and the enhanced thrombolysis mediated by magnetic tPA-MRs under an extra rotational magnetic field. (3) Recanalization of blood flow in the distal middle cerebral artery.

of rt-PA only. In clinical trials, Molina et al. [83] showed stroke patients who received a treatment strategy composed of galactose-based microbubbles, rt-PA and ultrasound could get a better recanalization effect than the remainder who received rt-PA combined with ultrasound or not [83]. Another potential mechanism of thrombolysis improvement mediated by combination of microbubbles, $\mathrm{ul}$ trasound and rt-PA may be enhancement of rt-PA diffusion at the surface of the thrombus which is triggered by mechanical effect. Rare adverse effects have been reported in the current clinic application and it is widely considered safe.

\subsection{Metallic NPs and Magnetic NPs}

Metallic NPs are made up of shells on the surface and inorganic metallic or metallic oxide cores. While platinum NPs appear to show antioxidant effect by eliminating hydrogen peroxide [100], reactive oxygen species (ROS) scavenging properties are also presented by cerium oxide NPs [101]. Another kind of metallic NPs is magnetic NPs (MNPs), which contains a paramagnetic iron oxide core, made of magnetite $\left(\mathrm{Fe}_{3} \mathrm{O}_{4}\right)$ or maghemite $\left(\gamma-\mathrm{Fe}_{2} \mathrm{O}_{3}\right)$ or a mixture, and covered by a polymers shell. To date, magnetic iron oxide nanoparticles (MPIOs) have been widely explored, and they can be divided into different categories according to the diameter. Standard superparamagnetic iron oxides (SPIOs) particles with a mean diameter between 50 and a few hundred nanometers, ultrasmall SPIOs (USPIOs) with a diameter of 10-50 nanometers, and very small SPIOs (VSPIOs) with a diameter of less than 10 nanometers [102]. One advantage of magnetic nanoparticles in the thrombolytic therapy is related to their magnetic feature. Accompany with the local application of a magnetic field, magnetic nanoparticles that combine drugs tend to converge to a designated site [103]. The removal of MNPs from treticuloendothelial system (RES) depends mainly on the size, coating, and approach of delivery of the nanoparticles. Zhou et al. [84] developed PLGA nanocarriers loaded with rt-PA, and applied it to a rat model of ferric chlorideIn induced abdominal aortic thrombi successfully [84]. Although there are some weaknesses such as the low encapsulation efficacy and partial loss of rt-PA activity. Absar et al. [104] observed an enhancement of 35\% of thrombolytic effectiveness for rt-PA-loaded into PEGylated liposomes in rat experiment when compared with free rt-PA. Nedelmann et al. [82] found rt-PA loaded into microbubbles and with ultrasounds on assisted can restored the blood flow completely in MCAO rat model, while rt-PA alone improved partial perfusion [82]. Ma et al. [103] used t-PA-MNPs (equivalent to 20\% of t-PA dose) to restore $82 \%$ of iliac artery blood flow in rats within $75 \mathrm{~min}$. Cheng et al. [105] successfully constructed a magnetic nickel nanorods, which driven by a rotating magnetic field could significantly enhance the mechanical force compared to spherical MNPs.

\section{$4 \mathrm{t}$-PA conjugated MRs for ischemic stroke}

In the rat model of femoral artery thrombosis, nickel nanorods (NRs) can effectively improve the thrombolysis efficiency of t-PA in vivo and promote the recanalization of obstructed femoral artery. However, with the biological toxicity of nickel and the free t-PA is difficult to meet the requirements of targeted thrombolysis. Considering 
the limitation of nickel's native biological toxicity and inefficiency of free t-PA, Hu et al. [106] developed a nontoxic $\mathrm{Fe}_{3} \mathrm{O}_{4}$-microrods (MRs) subsequently. In vitro experiments confirmed that the thrombolysis rate was significantly increased once $\mathrm{t}-\mathrm{PA}-\mathrm{Fe}_{3} \mathrm{O}_{4}$-MRs was driven by a rotating magnetic field. The possible explanation for this result is the synergistic effect of local t-PA aggregation and mechanical agitation by rod. To further confirm the advanced thrombolysis effects of tPA-MRs, our group built a ferric chloride induced-middle cerebral occlusion model, which blood clot was formed in the middle cerebral artery. The results demonstrated that under the guidance of an external magnet, intra-arterial injection of tPA-MRs could target the cerebral blood clot in vivo, where tPA was subsequently released at the site of embolism. Meanwhile, with the application of an external rotating magnetic field, rotating magnetic MRs significantly improved the mass transport of the tPA-clot reaction, as well as the efficiency of mechanical disruption of the clot network (Figure 2, Figure S1). Importantly, intravenously injected MRs could be discharged from the kidney, and the function of liver and kidney were not impaired after administration of tPAMRs [2, 85].

\section{Conclusion}

Based on constant explorations, nanoparticulate drugdelivery systems hold great prospects in overcoming some barriers involved in stroke treatment. Nanocarries potentially open a new delivery approach targeting t-PA to the specific lesion site and dramatically enhance the thrombolysis efficiency, at the meantime, decrease the risk of ICH, expand the therapeutic time window and improve clinical outcomes. Nanocarriers also protect drugs from enzymatic degradation and accelerate targeted drug release while loading the maximum dose of drugs to ensure thrombolytic effect. Furthermore, novel nanomaterial such as USPIOs and VSPIOs will become a more appropriate material for nanothrombolysis in the future. From bench to clinic application, more in-depth and meaningful studies are needed.

Funding: This work was supported by the National Natural Science Foundation of China (No. 81771262), Zhejiang Health Science and Technology Project (2016RCA022), Zhejiang Key Research and Development Project (2017C03027), Sigma Xi Grants-in-Aid of Research Program Fellowship for Jiangnan Hu (G2017100192773410).

\section{References}

[1] V. L. Roger, A. S. Go, D. M. Lloyd-Jones, E. J. Benjamin, J. D. Berry, W. B. Borden, et al., Circulation,1 (2012) 188-197.

[2] C. Ren, N. Li, S. Li, R. Han, Q. Huang, J. Hu, et al., Aging Dis, 5 (2018) 869-879.

[3] G. A. Donnan, M. Fisher, M. Macleod, and S. M. Davis, Lancet, 9624 (2008) 1612-1623.

[4] G. Stoll, C. Kleinschnitz, and B. Nieswandt, Blood, 9 (2008) 35553562.

[5] C. S. Mang, K. L. Campbell, C. J. Ross, and L. A. Boyd, Phys Ther, 12 (2013) 1707-1716.

[6] G. B. D. DALYs, H. Collaborators, Lancet, 10159 (2018) 1859-1922.

[7] V. Kumar, A. K. Abbas, and J. C. Aster, Robbins and Cotran Pathologic Basis of Disease, 9th Edition, Philadelphia: Elsevier Saunders, (2015), pp. 1263.

[8] X. Xu, B. Wang, C. Ren, J. Hu, D. A. Greenberg, T. Chen, et al., Aging Dis, 5 (2017) 590- 610.

[9] X. Xu, B. Wang, C. Ren, J. Hu, D. A. Greenberg, T. Chen, et al., Aging Dis, 4(2017) 486- 505.

[10] C. Turesson, A. Jarenros, and L. Jacobsson, Ann Rheum Dis, 8 (2004) 952-955.

[11] J. Astrup, B. K. Siesjo, L. Symon, Stroke, 6 (1981) 723-725.

[12] C. Ren, Y. Yao, R. Han, Q. Huang, H. Li, B. Wang, et al., Exp Neurol, 304 (2018) 30-40.

[13] A. Bivard, L. Lin, M. W. Parsonsb, J Stroke, 2 (2013) 90-98.

[14] A. Meretoja, M. Keshtkaran, J. L. Saver, T. Tatlisumak, M. W. Parsons, M. Kaste, et al., Stroke,4 (2014) 1053-1058.

[15] T. Robinson, Z. Zaheer, and A. K. Mistri, Ther Adv Chronic Dis, 2 (2011) 119-131.

[16] E. C. Jauch, J. L. Saver, H. P. Adams, Jr., A. Bruno, J. J. Connors, B. M. Demaerschalk, et al., Stroke, 3 (2013) 870-947.

[17] J. H. Rha and J. L. Saver, Stroke, 3 (2007) 967-973.

[18] O. A. Berkhemer, P. S. Fransen, D. Beumer, L. A. van den Berg, H. F. Lingsma, A. J. Yoo, et al., N Engl J Med,1 (2015) 11-20.

[19] E. A. Samaniego, G. Dabus, and I. Linfante, Front Neurol, 2 (2011) 76.

[20] National Institute of Neurological Disorders and Stroke rt-PA Stroke Study Group, N Engl J Med, 333(24) (1995) 1581-1588.

[21] A. M. Thiebaut, M. Gauberti, C. Ali, S. Martinez De Lizarrondo, D. Vivien, M. Yepes, et al., Lancet Neurol,12 (2018) 1121-1132.

[22] W. Hacke, M. Kaste, E. Bluhmki, M. Brozman, A. Davalos, D. Guidetti, et al. N Engl J Med, 13 (2008) 1317-1329.

[23] N. Wahlgren, N. Ahmed, A. Davalos, W. Hacke, M. Millan, K. Muir, et al., Lancet, 9646 (2008) 1303-1309.

[24] The European Stroke Organisation (ESO) Executive Committee and the ESO Writing Committee, Cerebrovasc Dis, 25 (2008) 457507.

[25] M. T. Torbey, E. Jauch, D. S. Liebeskind, and Acute Stroke Advisory Board of the National Stroke, N Engl J Med, 259 (26) (2008) 2839; author reply 2841 .

[26] J. Emberson, K. R. Lees, P. Lyden, L. Blackwell, G. Albers, E. Bluhmki, et al., Lancet, 9958 (2014) 1929-1935.

[27] I. S. T. c. group, P. Sandercock, J. M. Wardlaw, R. I. Lindley, M. Dennis, G. Cohen, et al., Lancet, 9834 (2012) 2352-2363.

[28] R. El Khoury, R. Jung, A. Nanda, C. Sila, M. G. Abraham, A. C. Castonguay, et al., Neurology, 79 (13 Suppl 1) (2012) S26-34.

[29] J. P. Broderick, Y. Y. Palesch, A. M. Demchuk, S. D. Yeatts, P. Khatri, M. D. Hill, et al., N Engl J Med,10 (2013) 893-903. 
[30] C. S. Kidwell, R. Jahan, J. Gornbein, J. R. Alger, V. Nenov, Z. Ajani, et al., N Engl J Med,10 (2013) 914-923.

[31] T. Bonnard, M. Gauberti, S. Martinez de Lizarrondo, F. Campos, and D. Vivien, Stroke, 5 (2019) 1318-1324.

[32] D. Collen, D. C. Rijken, J. Van Damme, and A. Billiau, Thromb Haemost, 3 (1982) 294-296.

[33] D. Pennica, W. E. Holmes, W. J. Kohr, R. N. Harkins, G. A. Vehar, C. A. Ward, et al., Nature, 5897 (1983) 214-221.

[34] J. R. Haeberle, T. A. Sutton, and B. A. Trockman, J Biol Chem, 9 (1988) 4424-4429.

[35] A. Chevilley, F. Lesept, S. Lenoir, C. Ali, J. Parcq, D. Vivien, Front Cell Neurosci, 9 (2015) 415.

[36] K. Benchenane, V. Berezowski, C. Ali, M. Fernandez-Monreal, J. P. Lopez-Atalaya, J. Brillault, et al., Circulation, 17 (2005) 22412249.

[37] J. P. Lopez-Atalaya, B. D. Roussel, D. Levrat, J. Parcq, O. Nicole, Y. Hommet, et al., J Cereb Blood Flow Metab, 6 (2008) 1212-1221.

[38] C. Thelwell and C. Longstaff, J Thromb Haemost, 4 (2007) 804811.

[39] A. Mehra, C. Ali, J. Parcq, D. Vivien, and F. Docagne, Biochim Biophys Acta, 3 (2016) 395- 402.

[40] T. Boeckh-Behrens, J. F. Kleine, C. Zimmer, F. Neff, F. Scheipl, J. Pelisek, et al., Stroke, 7 (2016) 1864-1871.

[41] P. B. Sporns, U. Hanning, W. Schwindt, A. Velasco, J. Minnerup, T. Zoubi, et al., Stroke, 8 (2017) 2206-2210.

[42] P. Bhattacharjee and D. Bhattacharyya, An Insight into the Abnormal Fibrin Clots - Its Pathophysiological Roles edited by K Kolev, Rijeka: Fibrinolysis and Thrombolysis: InTechOpen, (2014), pp. 1-29.

[43] M. Yepes, M. Sandkvist, E. G. Moore, T. H. Bugge, D. K. Strickland, and D. A. Lawrence, J Clin Invest, 10 (2003) 1533-1540.

[44] O. Nicole, F. Docagne, C. Ali, I. Margaill, P. Carmeliet, E. T. MacKenzie, et al., Nat Med, 1 (2001) 59-64.

[45] A. M. Martin, C. Kuhlmann, S. Trossbach, S. Jaeger, E. Waldron, A. Roebroek, et al., J Biol Chem, 18 (2008) 12004-12013.

[46] E. Lemarchand, E. Maubert, B. Haelewyn, C. Ali, M. Rubio, and D. Vivien, Cell Death Differ,1 (2016) 123-131.

[47] A. Montagne, M. Hebert, A. Jullienne, F. Lesept, A. Le Behot, M. Louessard, et al., Stroke, 10 (2012) 2774-2781.

[48] F. Wu, R. Echeverry, J. Wu, J. An, W. B. Haile, D. S. Cooper, et al., Mol Cell Neurosci, 52 (2013) 9-19.

[49] W. B. Haile, J. Wu, R. Echeverry, F. Wu, J. An, and M. Yepes, J Cereb Blood Flow Metab,1 (2012) 57-69.

[50] R. J. Turner and F. R. Sharp, Front Cell Neurosci, 10 (2016) 56.

[51] M. Yepes, B. D. Roussel, C. Ali, and D. Vivien. Trends Neurosci, 1 (2009) 48-55.

[52] J. Yang, S. Ding, W. Huang, J. Hu, S. Huang, Y. Zhang, et al., Front Neurosci, 10 (2016) 61.

[53] W. Hacke, M. Kaste, C. Fieschi, D. Toni, E. Lesaffre, R. von Kummer, et al., JAMA, 13 (1995) 1017-1025.

[54] W. Hacke, M. Kaste, C. Fieschi, R. von Kummer, A. Davalos, D. Meier, et al., Lancet, 9136 (1998) 1245-1251.

[55] W. M. Clark, S. Wissman, G. W. Albers, J. H. Jhamandas, K. P. Madden, and S. Hamilton, Jama, 21 (1999) 2019-2026.

[56] W. Hacke, G. Donnan, C. Fieschi, M. Kaste, R. von Kummer, J. P. Broderick, et al., Lancet, 9411 (2004) 768-774.

[57] X. Huang, B. K. Cheripelli, S. M. Lloyd, D. Kalladka, F. C. Moreton, A. Siddiqui, et al., Lancet Neurol, 4 (2015) 368-376.

[58] M. Parsons, N. Spratt, A. Bivard, B. Campbell, K. Chung, F. Miteff, et al., N Engl J Med, 12 (2012) 1099-1107.
[59] J. M. Wardlaw, V. Murray, E. Berge, and G. J. del Zoppo, Cochrane Database Syst Rev, 7 (2014) CD000213.

[60] V. Larrue, R. von Kummer, G. del Zoppo, and E. Bluhmki, Stroke, 5 (1997) 957-960.

[61] M. J. Reeves, S. Arora, J. P. Broderick, M. Frankel, J. P. Heinrich, S. Hickenbottom, et al., Stroke, 6 (2005) 1232-1240.

[62] C. Longstaff and C. Thelwell, FEBS Lett, 15 (2005) 3303-3309.

[63] C. A. Cronin, J Emerg Med, 1 (2010) 99-105.

[64] L. Davydov and J. W. Cheng, Clin Ther, 7 (2001) 982-997 (discussion 981).

[65] E. C. Haley Jr., P. D. Lyden, K. C. Johnston, T. M. Hemmen, TNK in Stroke Investigators, Stroke, 3 (2005) 607-612.

[66] W. Hacke, G. Albers, Y. Al-Rawi, J. Bogousslavsky, A. Davalos, M. Eliasziw, et al., Stroke, 1 (2005) 66-73.

[67] A. J. Furlan, D. Eyding, G. W. Albers, Y. Al-Rawi, K. R. Lees, H. A. Rowley, et al., Stroke, 5 (2006) 1227-1231.

[68] W. Hacke, A. J. Furlan, Y. Al-Rawi, A. Davalos, J. B. Fiebach, F. Gruber, et al., Lancet Neurol, 2 (2009) 141-150.

[69] C. S. Kidwell, L. Latour, J. L. Saver, J. R. Alger, S. Starkman, G. Duckwiler, et al., Cerebrovasc Dis, 4 (2008) 338-343.

[70] A. Kassner, T. P. Roberts, B. Moran, F. L. Silver, and D. J. Mikulis, AJNR Am J Neuroradiol, 10 (2009) 1864-1869.

[71] E. J. Su, L. Fredriksson, M. Geyer, E. Folestad, J. Cale, J. Andrae, et al., Nat Med, 7 (2008) 731-737.

[72] K. Benchenane, J. P. Lopez-Atalaya, M. Fernandez-Monreal, O. Touzani, and D. Vivien, Trends Neurosci, 3 (2004) 155-160.

[73] T. H. Mouhieddine, M. M. Itani, A. Nokkari, C. Ren, G. Daoud, A. Zeidan, et al., Curr Neurol Neurosci Rep, 1 (2015) 505.

[74] Y. Yoshioka, T. Yoshikawa, and Y. Tsutsumi, Nihon Eiseigaku Zasshi, 65(4) (2010) 487-492.

[75] X. Hua, L. Zhou, P. Liu, Y. He, K. Tan, Q. Chen, et al., J Thromb Thrombolysis, 1 (2014) 57-64.

[76] A. O. Elzoghby, M. M. Abd-Elwakil, K. Abd-Elsalam, M. T. Elsayed, Y. Hashem, and O. Mohamed, Curr Pharm Des, 22 (2016) 33053323.

[77] L. Huang, J. Hu, S. Huang, B. Wang, F. Siaw-Debrah, M. Nyanzu, et al., Prog Neurobiol, 157 (2017) 29-48.

[78] J. L. Heeremans, R. Prevost, M. E. Bekkers, P. Los, J. J. Emeis, C. Kluft, et al., Thromb Haemost, 3 (1995) 488-494.

[79] J.-Y. Kim, J.-K. Kim, J.-S. Park, Y. Byun, and C.-K. Kim, Biomaterials, 29 (2009) 5751-5756.

[80] T. W. Chung, S. S. Wang, and W. J. Tsai, Biomaterials, 2 (2008) 228-237.

[81] Y. Uesugi, H. Kawata, J. Jo, Y. Saito, and Y. Tabata, J Control Release, 2 (2010) 269-277.

[82] M. Nedelmann, N. Ritschel, S. Doenges, A. C. Langheinrich, T. Acker, P. Reuter, et al., J Cereb Blood Flow Metab, 10 (2010) 17121720.

[83] C. A. Molina, M. Ribo, and M. Rubiera, J. Montaner, E. Santamarina, R. Delgado-Mederos, et al., Stroke, 2 (2006) 425-429.

[84] J. Zhou, D. Guo, Y. Zhang, W. Wu, H. Ran, and Z. Wang, ACS Appl Mater Interfaces, 8 (2014) 5566-5576.

[85] J. Hu, S. Huang, L. Zhu, W. Huang, Y. Zhao, K. Jin, et al., ACS Appl Mater Interfaces, 39 (2018) 32988-32997.

[86] B. Vaidya, M. K. Nayak, D. Dash, G. P. Agrawal, and S. P. Vyas., Int J Pharm,1-2 (2011) 254-261.

[87] S. L. Huang, Adv Drug Deliv Rev, 10 (2008) 1167-1176.

[88] T. Nii and F. Ishii, Int J Pharm, 1 (2005) 198-205.

[89] C. Psarros, R. Lee, M. Margaritis, and C. Antoniades. Nanomedicine, 8 (Suppl. 1) (2012) S59-68. 
[90] I. M. El-Sherbiny, I. E. Elkholi, and M. H. Yacoub, Glob Cardiol Sci Pract, 3 (2014) 336-349.

[91] J. Y. Kim, J. K. Kim, J. S. Park, Y. Byun, and C. K. Kim, Biomaterials, 29 (2009) 5751-5756.

[92] X. Zhang, D. Y. Alakhova, E. V. Batrakova, S. Li, Z. Yang, Y. Li, et al., J Neuroimmune Pharmacol, 1 (2009) 35-46.

[93] S. V. Bhujbal, P. de Vos, and S. P. Niclou, Adv Drug Deliv Rev, 67-68 (2014) 142-153.

[94] C. E. Astete and C. M. Sabliov, J Biomater Sci Polym Ed, 3 (2006) 247-289.

[95] A. Mdzinarishvili, V. Sutariya, P. K. Talasila, W. J. Geldenhuys, and P. Sadana, Drug Deliv Transl Res, 4 (2013) 309-317.

[96] X. Liu, C. An, P. Jin, X. Liu, and L. Wang, Biomaterials, 3 (2013) 817-830.

[97] N. Kamaly, B. Yameen, J. Wu, and O. C. Farokhzad. Chem Rev, 4 (2016) 2602-2663.

[98] M. De Saint Victor, C. Crake, C. C. Coussios, E. Stride, Expert Opin Drug Deliv, 2 (2014) 187-209.
[99] A. Moumouh, L. Barentin, F. Tranquart, S. Serrierre, I. Bonnaud, and J. P. Tasu, Ultrasound Med Biol, 1 (2010) 51-57.

[100] M. Takamiya, Y. Miyamoto, T. Yamashita, K. Deguchi, Y. Ohta, and K. Abe. Neuroscience, 221 (2012) 47-55.

[101] M. Das, S. Patil, N. Bhargava, J. F. Kang, L. M. Riedel, S. Seal, et al., Biomaterials, 10 (2007) 1918-1925.

[102] J. S. Weinstein, C. G. Varallyay, E. Dosa, S. Gahramanov, B. Hamilton, W. D. Rooney, et al., J Cereb Blood Flow Metab, 1 (2010) 15-35.

[103] Y. H. Ma, S. Y. Wu, T. Wu, Y. J. Chang, M. Y. Hua, J. P. Chen., Biomaterials, 19 (2009) 3343-3351.

[104] S. Absar, K. Nahar, Y. M. Kwon, and F. Ahsan, Pharm Res, 6 (2013) 1663-1676.

[105] R. Cheng, W. Huang, L. Huang, B. Yang, L. Mao, K. Jin, et al., ACS Nano, 8 (2014) 7746-7754.

[106] J. Hu, W. Huang, S. Huang, Q. ZhuGe, K. Jin, and Y. Zhao, Nano Research, 9 (2016) 2652-2661. 